\title{
LBV (candidate) nebulae: bipolarity and outflows
}

\author{
Kerstin Weis ${ }^{1}$ \\ Max-Planck-Institut für Radioastronomie, \\ Auf dem Hügel 69, D-53121 Bonn, BRD, \\ and \\ Department of Astronomy, University of Minnesota, \\ 116 Church Street SE, Minneapolis, MN 55455, USA
}

\begin{abstract}
The most massive evolved stars (above $50 \mathrm{M}_{\odot}$ ) undergo a phase of extreme mass loss, in which their evolution is reversed from a redward to a blueward motion in the H-R diagram. In this phase, the stars are known as Luminous Blue Variables and they are located in the H-R diagram close to the Humphreys-Davidson limit. It is far from understood what causes the strong mass loss or what triggers the so-called giant eruptions, active events in which in a short time a large amount of mass is ejected. Here I present results from a larger project, devoted to better understand LBVs through studying the LBV nebulae. These nebulae are formed as a consequence of the strong mass loss. The analysis concentrates on the morphology and kinematics of these nebulae. Of special concern is the frequently observed bipolar nature of the LBV nebulae. Bipolarity seems to be a general feature and strongly constrains models of the LBV phase, especially of the formation of the nebulae. In addition, we find outflows from LBV nebulae, the first evidence for ongoing instabilities in the nebulae.
\end{abstract}

\section{LBV nebulae - their morphology and kinematics}

To better understand why massive stars in the LBV phase increase their mass loss and what triggers the sporadic and powerful giant eruptions, we study the LBV nebulae, obvious relics of these processes. Using high-resolution images and high-dispersion echelle spectra, we concentrate on the morphology and kinematics of all LBV nebulae which are resolvable by HST. This limits our sample to nebulae in the Milky Way, LMC and SMC. For a more detailed description of the results and individual objects, see Weis et al. (1997), Weis (2000), Weis (2001a,b), Weis \& Duschl (2002), Weis et al. (submitted), and Weis (in preparation). Reviews on LBVs and LBV nebulae are presented by Humphreys \& Davidson (1994), Nota et al. (1995), Weis (2001b) and references therein. Here I summarize our global results (Weis 2001b, for references on Table 1). Sizes and expansion velocities of LBV nebulae are given in Table 1.

\footnotetext{
${ }^{1}$ Feodor-Lynen-fellow, Alexander-von-Humboldt foundation
} 
Table 1. Parameters of known LBV nebulae.

\begin{tabular}{llccl}
\hline \hline $\begin{array}{l}\text { LBV } \\
\text { (candidate) }\end{array}$ & host galaxy & $\begin{array}{c}\text { size } \\
(\mathrm{pc})\end{array}$ & $\begin{array}{c}v_{\text {exp }} \\
\left(\mathrm{km} \mathrm{s}^{-1}\right)\end{array}$ & morphology \\
\hline$\eta$ Car & Milky Way & $0.2 / 0.67$ & $600 / 10-2000$ & bipolar \\
HR Car & Milky Way & $1.3 \times 0.65$ & $75-150$ & bipolar \\
P Cyg & Milky Way & $0.2 / 0.8$ & $110-140 / 185$ & spherical/clumpy \\
AG Car & Milky Way & $0.87 \times 1.16$ & 70 & bipolar (?) \\
Wray 751 & Milky Way & 0.5 & 26 & spherical/bipolar \\
He 3-519 & Milky Way & 2.1 & 61 & spherical (?) \\
HD 168625 & Milky Way & $0.13 \times 0.17$ & 40 & spherical/clumpy \\
Pistol Star & Milky Way & $0.8 \times 1.2$ & 60 & spherical/clumpy \\
R 127 & LMC & 1.3 & 32 & spherical/bipolar \\
R 143 & LMC & 1.2 & $24(?)$ & clumps \\
S 61 & LMC & 0.82 & 27 & spherical/outflow (?) \\
S 119 & LMC (?) & 1.8 & 26 & spherical/outflow \\
\hline Sher 25 (?) & Milky Way & 1 & 70 & bipolar \\
Sk-69 $279(?)$ & LMC & 4.5 & 14 & spherical/outflow \\
\hline \hline
\end{tabular}

Among LBV nebulae $\eta$ Car seems special. It shows the highest expansion velocities, $2000 \mathrm{~km} \mathrm{~s}^{-1}$ (see Weis et al. in these Proceedings), found among LBV nebulae and its shape is highly bipolar. The expansion velocity is unique (Table 1), but the morphology not! HR Car (Weis et al. 1997) is bigger in size, but with the same bipolar structure. At the same time, the expansion velocity is lower, indicating that HR Car might be an older ( $4000-9000 \mathrm{yr})$, sloweddown version of $\eta$ Car's nebula. Other nebulae (e.g., Wray 751, R 127) are not as strong bipolar, but still show components which move bi-directional. Finally, some nebulae are spherical, but reveal a very prominent outflow feature (e.g., $\mathrm{S} 119, \mathrm{Sk}-69^{\circ} 279$, a new LBV candidate). In summary, we find that instabilities in LBV nebulae are present and that the bipolarity occurs frequently, but with different strength. At this stage we can only speculate whether or not it might be due to stellar rotation, asymmetrical/bipolar winds or binarity - or something we have not thought of yet. Nevertheless, a new constraint to the evolution of LBVs is that bipolarity has to be achieved while forming these nebulae.

\section{References}

Humphreys, R.M., Davidson, K. 1994, PASP 106, 1025

Nota, A., Livio, M., Clampin, M., Schulte-Ladbeck, R.E. 1995, ApJ 448, 788

Weis, K., Duschl, W.J., Bomans, D.J., Chu, Y.-H., Joner, M.D. 1997, A\&A 320, 568

Weis, K. 2000, A\&A 357, 938

Weis, K. 2001a, in: T.R. Gull, S. Johansson \& K. Davidson (eds.), Eta Carinae and Other Mysterious Stars, ASP-CS 242, 129

Weis, K. 2001b, in: R.E. Schielicke (ed.), Reviews in Mod. Astron. 14, 261

Weis, K., Duschl, W.J. 2002, A\&A 393, 503 